\title{
FEATURES OF THE INFLUENCE OF THERMAL MODES OF COOLING ON PROCESSES OF NONEQUILIBRIUM CRYSTALLIZATION OF MATERIALS FROM THE LIQUID STATE
}

\author{
V.F. Bashev*, S.I. Ryabtsev, S.N. Antropov, N.A. Kutseva, O.I. Kushnerov, \\ E.S. Skorbyaschensky \\ Oles Honchar Dnipro National University, Dnipro, Ukraine \\ *e-mail: bashev_vf@ukr.net
}

\begin{abstract}
The quantitative estimation of maximum level of cooling rates in the process of casting microwires in glass insulation is given. The shown possibility of nonequilibrium formation of microwire substance is due to the influence of an amorphous substrate in the form of glass insulation. The amorphous state in the case of thin microwires with cast iron vein $\mathrm{Fe}-20$ at.\% $\mathrm{C}$ confirms the implementation of an increased (compared to splat-quenching) level of nonequilibrium formation of microwires in combination with updated rates of cooling and increased degree of supercooling of liquid microwire vein.

Keywords: nonequilibrium cooling, cast microwire; crystallization, splat-quenching.
\end{abstract}

Received 09.11.2018; Received in revised form 7.12.2018; Accepted 21.12.2018

\section{Introduction}

In the process of forming samples with required properties under conditions of nonequilibrium cooling, it is necessary to create such technological modes of crystallization, in which the necessary cooling rate (c.r.) of material would be realized. The latter determines, first of all, the formation of both morphology and a number of physical-chemical properties rapid-quenched films and cast microwires (MW) in glass insulation. Methods of direct experimental determination of cooling rates in such samples are practically absent; therefore it is necessary to obtain design ratios using some provisions of the general theory of heat transfer.

\section{Materials and methods}

In the process of heat transfer, the heat exchange occurs mainly through the collision of the melt and cooling medium, which is described by the well-known Newton's equation [1]:

$$
q=\alpha\left(T_{P}-T_{c}\right)
$$

where $q$ is specific heat flux; $\alpha$ is a coefficient of heat transfer, $\left(T_{P}-T_{C}\right)$ is the difference between the temperature of the wall and the cooling medium.

To describe the thermal processes in the splat-quenching (s.q.) method on the inner surface of a rapidly rotating cylinder, a one-way heat flux through a film-cylinder contact surface is used with applying the Fourier heat conduction equation. To simplify the calculations, it was assumed that the thermal flux through the free surface of the film can be neglected [1]. Incidentally, there are experimental results, which imply that in some cases one can observe simultaneous crystallization on the contact and free surface [2]. In the last work by the authors, when obtaining $\mathrm{Fe}_{76.1} \mathrm{Nb}_{3.0} \mathrm{Cu}_{1.0} \mathrm{Si}_{13.8} \mathrm{~B}_{6.1}$ metal glass, a computer modeling of surface crystallization processes due to heat transfer through the free surface depending on the quenching modes was carried out. It was found that depending on the nature of the nucleation of crystals and the rate of growth, it was possible to actualize all the variegated phenomena of surface crystallization, which are observed in the preparation of metallic glass.

Therefore, under these conditions the assessment of c.r. on a free surface of the film and vein of MW in glass isolation (obtained by the Ulitovskii-Tailor method) $[3,4]$ is of particular interest. First of all, the difficulty of estimating the c.r. is connected with the ascertainment of the dependence of the heat transfer coefficient $\alpha$ on parameters that determine it. 
It is experimentally established [1] that $\alpha$ depends on the diameter of MW, film size, thermophysical properties of the cooling medium, and temperature difference $\left(T_{P}-T_{C}\right)$. The properties of the medium are described by the following basic parameters: the coefficient of thermal expansion $\beta\left[\mathrm{K}^{-1}\right]$, thermal conductivity $\lambda[\mathrm{W} /(\mathrm{m}$ $\mathrm{K})]$, specific heat $[\mathrm{J} /(\mathrm{kg} \mathrm{K})]$, density $\rho$, dynamic and kinematic viscosities $\mu$ and $\nu=\mu / \rho$ $\left[\mathrm{m}^{2} / \mathrm{s}\right]$, temperature conductivity $a[\mathrm{~kg} /(\mathrm{m} \mathrm{s})]$ and a common parameter that depends on the shape, structure of the surface and its size. Getting this kind of experimental dependencies is practically unrealistic due to a large number of parameters that affects the process under study. That is why relations obtained using the similarity theory [1] and some experimental dependencies were applied in the work. All the parameters that influence the thermal conductivity can be described in three thermophysical complexes: the number of Nusselt $(\mathrm{Nu})$, the number of Grashof $(\mathrm{Gr})$, Prandtl number $(\mathrm{Pr})$

$$
N u=\alpha L / \lambda ; G r=\beta g L^{3}\left(T-T_{C}\right) / v^{2} ; \operatorname{Pr}=v / a, a=\lambda / c_{P} \rho
$$

where $L$ is geometric size characteristic for the body of this configuration; $g$ is acceleration of gravity.

It is convenient to determine the coefficient of heat transfer from the criterion equation:

$$
N u=F(G r, \operatorname{Pr}) .
$$

For the average heat output when cooled in the air, the last equation has the form:

$$
N u=0,037 \operatorname{Re}^{0,8} \operatorname{Pr}^{0,43}\left(\operatorname{Pr}_{1} / \operatorname{Pr}_{2}\right)^{0,25}
$$

where $\operatorname{Pr}_{1}, \operatorname{Pr}_{2}$ are Prandtl numbers at the film and environment temperatures.

The transformation of equation (2) gives the following expression for the coefficient of heat transfer $\alpha^{\prime}$ :

$$
\alpha^{\prime}=0,037(V d / v)^{0,8} 0,7^{0,43}(0,7 / 0,72)^{0,25} \lambda / d
$$

The experimentally obtained value of heat transfer coefficient on the boundary between the metal thermocouple with a diameter of $50 \mathrm{~mm}$ and running cool water is 4,2 $\mathrm{W} /\left(\mathrm{cm}^{2} \mathrm{~K}\right)$ [5]. However, the technical limitations of experiments did not allow determining the dependence of heat transfer coefficient on the diameter of MW and the volume change of cooling fluid. Because of the complexity of experiments for determining $\alpha$, there is a need for its theoretical calculation.

\section{Results and discussion}

In the case of infinite cylinders, we can apply the following formula for the convective coefficients of heat transfer for the practical use [1]:

$$
\alpha_{1}=A_{1}\left(\frac{T-T_{C}}{d^{5}}\right)^{1 / 8}
$$

where $d$ is the diameter of MW; $A_{1}$ is the coefficient taking into account the influence of physical parameters of the environment:

$$
A_{1}=1,18\left(\frac{\beta g v}{a}\right)^{1 / 8} \frac{\lambda_{m}}{v_{m}^{1 / 4}} .
$$




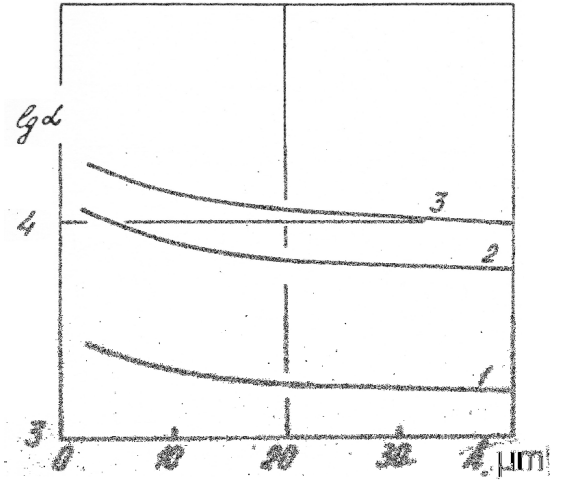

Fig. 1. Dependence of $\alpha$, for free surface films $\mathrm{Fe}-20$ at.\% $\mathrm{C}$, on the thickness and rotation: $1-70 \mathrm{~m} / \mathrm{s} ; 2-500 \mathrm{~m} / \mathrm{s} ; 3-1000 \mathrm{~m} / \mathrm{s}$.

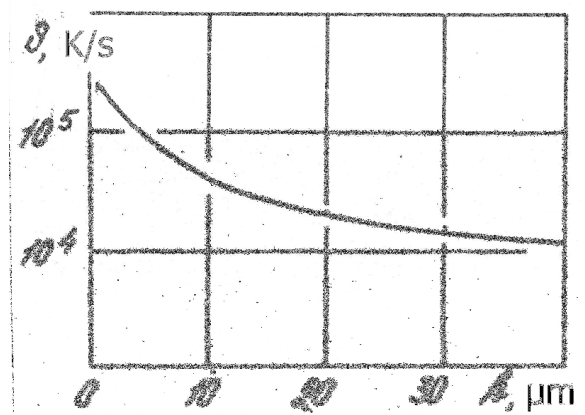

Fig. 2. Cooling rate of free surface of $\mathrm{Fe}-20$ at.\% $\mathrm{C}$ films (linear speed of rotation cylinder $-70 \mathrm{~m} / \mathrm{s}$ ).

The coefficients of heat transfer of MW during cooling in different cooling media are calculated below. To describe the cooling process of MW in the air, the equation for coefficients of heat transfer $\alpha_{1}$ was used [1]:

$$
\alpha_{1}(d)=\lambda_{1} \frac{0,26\left(\omega \frac{d}{v_{1}}\right)^{0,6}}{d}
$$

where $\lambda_{1}, v_{1}$ are thermal conductivity and air viscosity; $\omega, d$ are pull rate and MW diameter.

Resulting heat transfer coefficients at cooling MW in water and air are shown in Fig. 3.

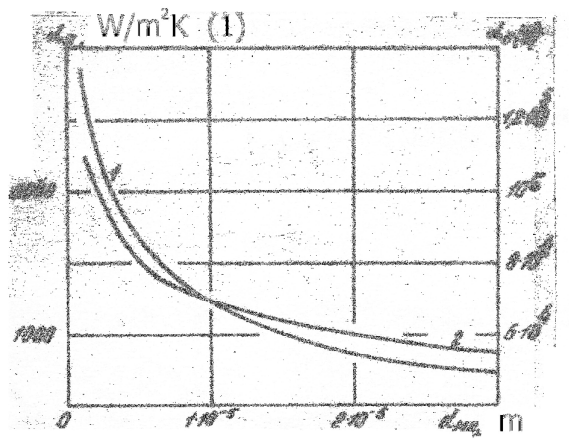

Fig. 3. Dependence of heat transfer coefficient on the wire diameter during cooling MW in air (1) and with water (2).

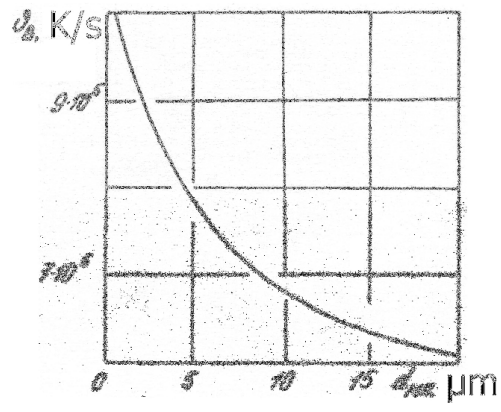

Fig. 4. Dependence of c.r. of MW on the wire diameter (cooling by water).

It is natural that the coefficient of water cooling is much higher, therefore the Nusselt criterion was calculated from the following equation:

$$
N u(d)=0,25\left(\omega \frac{d}{v_{2}}\right)^{0,8} \operatorname{Pr}_{2}{ }^{0,43}\left(\operatorname{Pr}_{2} / \operatorname{Pr}_{3}\right)^{0,25}
$$

where $\lambda_{2}, v_{2}$-thermal conductivity and water viscosity; $\operatorname{Pr}_{2}, \operatorname{Pr}_{3}$ are the Prandtl numbers of water column at saturation and the previous environment, respectively. 
For MW of large radii, the coefficient $\alpha$, depending on the thickness of the isolation, changes slightly and corresponds to the experimental value. However, with a decrease in the thickness of MW it sharply increases and for very thin specimens reaches $\sim 10^{5}$ $\mathrm{W} /\left(\mathrm{m}^{2} \mathrm{~K}\right)$ (Fig. 3).

The formation of crystals on a conductive substrate (Fig. 5) has several structural zones: columnar (on the boundary with a substrate) and zone of equilibrium crystals [5]. From the above microstructures (Fig.5, 6 ) it is evident that with the advancement of the first dendrites to the center of s.q.-films dendrites of the second order begin to form. Qualitative features of structuring under the conditions of large c.r. can be explained by the analysis of kinetics of crystallization and thermal cooling modes: first of all, the value of the Bio parameter (Bio criterion equals to $\alpha \delta / \lambda$ where $\alpha, \delta$, and $\lambda$ are the heat transfer coefficient, half thickness of s.q.-film, and heat conductivity, respectively). It is natural that on the surface of the film on the border with the thermal conductive medium supercooling is more than in its inner layers. Therefore, the conditions for the appearance of crystals on the substrate are more pertinent taking into account the additional possibility of the nucleation action of the substrate itself. Increasing of c.r. eliminates diffusion (concentration) supercooling, and the direction of nucleus growth that occurs at the depth of the liquid may not coincide with the direction of the external heat transfer (Fig. 6.).

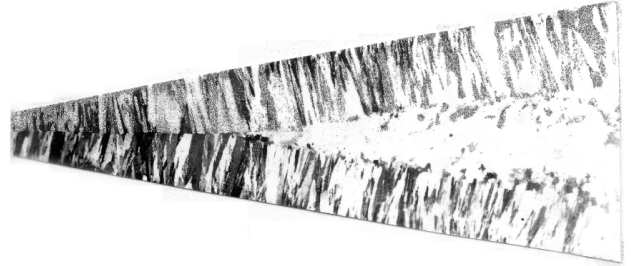

Fig. 5. Microstructure $(\times 300)$ of steel P-18 obtained in wedge casting form.

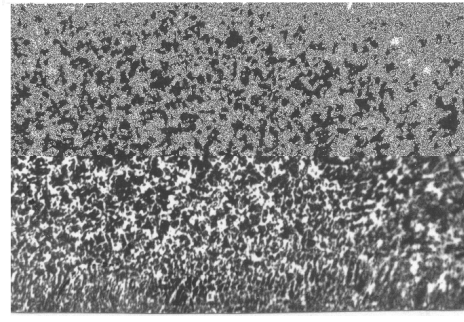

Fig.6. Microstructure of P-18 film, s.q. $\left(10^{6} \mathrm{~K} / \mathrm{c}\right), \times 1000$.

In order to explain quantitatively the emerging change in structural morphology, the problem of one-dimensional cooling of the medium with constant temperatures on the contact and free surface of the film with a thickness $\delta$ is solved. It is taken into account that the cooling process through the contact and free surface is independent. The general solution of Fourier differential equation

$$
\frac{\partial \theta}{\partial \tau}=a \frac{\partial^{2} \theta}{\partial x^{2}}
$$

is a sum of two opposite processes. It is accepted that the boundary conditions are:

a) $\mathrm{x}=0,\left(\frac{\partial \theta}{\partial x}\right)_{x=0}=0 ;$ б) $\mathrm{x}=\delta,\left(\frac{\partial \theta}{\partial x}\right)_{x=\delta}=-\frac{\alpha}{\lambda} \theta_{x=\delta}$. Initial conditions: at $\tau=0$ the temperature $\theta=\theta_{0}$.

The general solution of the Fourier equation with accounting of boundary conditions has a well-known form [11]:

$$
\theta=\sum_{n=1}^{n \rightarrow \infty} \frac{2 \theta_{0} \sin \mu_{n}}{\mu_{n}+\sin \mu_{n} \cos \mu_{n}} \cos \left(\mu_{n} \frac{x}{\delta}\right) \exp \left(-\frac{\mu_{n}^{2} a \tau}{\delta^{2}}\right)
$$


where $\mu_{1}, \mu_{2}, \ldots \mu_{n}$ are the solutions of equations $\operatorname{ctg} \mu_{n}=\mu_{n} / B i \quad(B i=\alpha \delta / \lambda)$; $a, \lambda$ are, respectively, temperature conductivity and thermal conductivity of films.

Using the dimensionless quantities $x / \delta=X(0 \leq X \leq 1), \theta_{n} / \theta_{0}=\theta$,

$F=a \tau / \delta^{2}$ (Fourier number), one can calculate the temperature distribution for the cross section of films of different thicknesses for the contact and free surface (Fig. 7, 8).

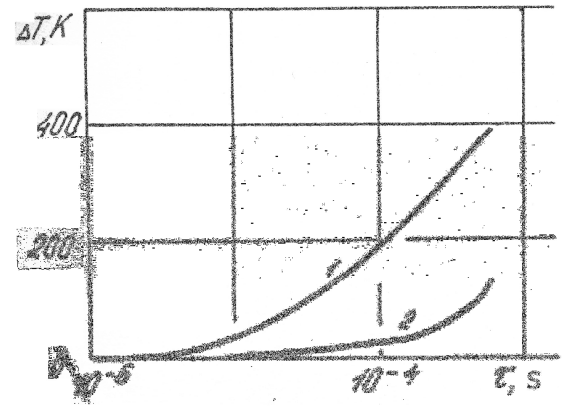

Fig. 7. Dependence of the temperature difference on time on the cross section of iron film $(\delta=10 \mu \mathrm{m})$ ? $\mu \mathbf{m})$ :

1,2 - for contact and free surface, respectively.

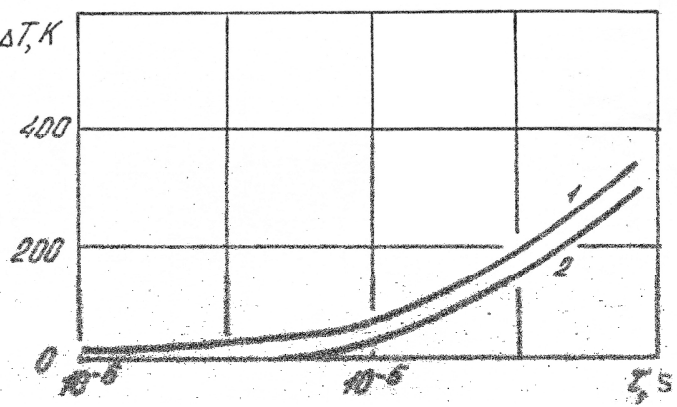

Fig. 8. Dependence of temperature difference on time on the cross section of iron film $(\delta=100$ 1,2 - for contact and free surface, respectively.

Table 1

\begin{tabular}{|c|c|c|c|c|c|c|}
\hline$\delta, 10^{-6} \mathrm{~m}$ & $\alpha_{K}, \mathrm{~W} / \mathrm{m}^{2} \mathrm{~K}$ & $\alpha_{C B}, \mathrm{~W} / \mathrm{m}^{2} \mathrm{~K}$ & $a, 10^{-6} \mathrm{~m}^{2} / \mathrm{s}$ & $\lambda, \mathrm{W} / \mathrm{mK}$ & $B i$ & $T, \mathrm{~K}$ \\
\hline 10 & $10^{5}$ & $10^{4}$ & 5 & 30 & 0,033 & 1810 \\
\hline 100 & $10^{5}$ & $10^{4}$ & 5 & 30 & 0,33 & 1810 \\
\hline
\end{tabular}

Notes: $\delta$ is a thickness of the film; $\alpha_{K}, \alpha_{C B}$ are coefficients of heat transfer on the contact and free surface of the film; $a$ is temperature conductivity; $\lambda$ is thermal conductivity; $B i$ is Bio criterion; $T$ is the temperature of the liquid film of iron on the eve of crystallization.

The dependences of this distribution on the contact and free surface of iron film with the heat transfer coefficients, respectively, $10^{5}$ and $10^{4} \mathrm{~W} /\left(\mathrm{m}^{2} \mathrm{~K}\right) \quad(B i=0,333)$ show qualitatively the observed changes in structural morphology of s.q.-films: at about one third of the distance from the free surface the supercooling value, which is the sum of both cooling processes (Fig. 9, 10), is the smallest. This may be the reason for the deviation from the direction of external heat transfer.

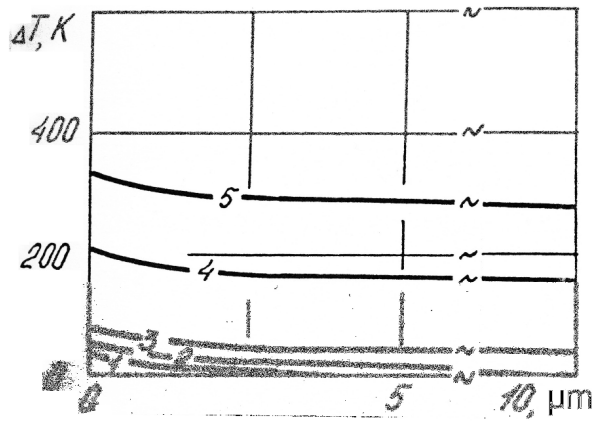

Fig. 9. Distribution of general supercooling in time for a film of $\mathrm{Fe}$ with thickness $10 \mu \mathrm{m}$.

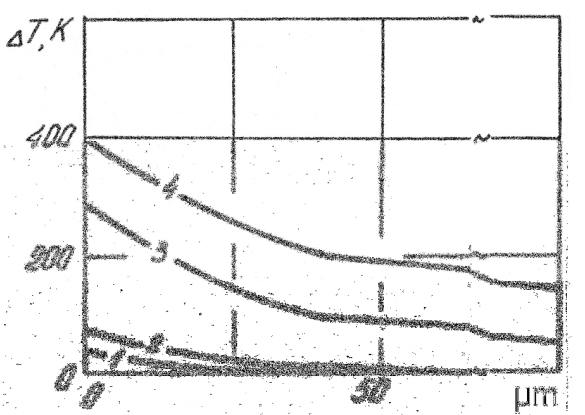

Fig. 10. Distribution of general supercooling in time for a film of Fe with thickness of $100 \mu \mathrm{m}$. 
The experimental confirmation of structural changes in the film morphology by the calculations of thermophysical cooling processes given above suggests that the proposed approach to determine the non-equilibrium cooling properties of films by the splat method is fairly correct.

\section{Conclusions}

1. Analysis of the thermal modes of cooling in the process of splat-quenching shows that there is simultaneous nonequilibrium crystallization on both (contact and free) sides of the film. The degrees of supercooling (respectively, 350 and $250 \mathrm{~K}$ ) and achieved cooling rates are sufficient for the nonequilibrium formation of the film structure. The features of nonequilibrium quenching from melt in the process of splat cooling can be found to provide a practical solution for the formation of amorphous-crystalline layers with a necessary set of improved physical properties in films.

2. The quantitative estimation of the maximum level of cooling rates in the process of micro-molding in glass insulation is given. Although this level is substantially lower than that achieved in the process of splat cooling, the possibility of more unbalanced formation of a substance in the case of MW is due to the influence of an amorphous substrate in the form of glass insulations. The amorphous state of thin MW with iron $\mathrm{Fe}-20$ at.\% C [6] confirms the implementation of the increased level of nonequilibrium formation of microwires in comparison with the splat-quenching in combination with updated cooling rates and increased degree of overcooling of liquid media of MW. The known experiments on overcooling moldings under a layer of broken glass, which passivates the catalytic action of constantly present in the melt impurities [6-9] and provides the absence of air between the mold and glass insulation, is the proof of reaching the great supercooling during the crystallization of liquid on the glass shell of MW.

\section{References}

1. Isachenko, V.P. Heat transfer / V.P. Isachenko, V.A. Osipova, A.S. Sukomel. M.: Energy, 1975. -485 p. Heat exchange, Aris, 2014. - 416p.

2. Volkov, V.A. About the mechanism of surface crystallization of metglasses at solidification of the melt on disk / V.A. Volkov, S.V. Pakhomov, V.I. Lad'yanov, A.V. Kulagin // Melts. - 1997. - N 5. - P.88 - 93.

3. Badinter, E.Ya Cast microwire and his properties / E.Ya. Badinter, I.F. Drabenko, B.I. Zaborovskii. - Kishineu: Shtiints, - 1973. - 318 p. (in Russian).

4. Baranov, S.A. Technology, preparation and properties of the cast glass-coated microwires / S.A Baranov, V.S. Larin, A.V. Torcunov // Crystals. - 2017. - Vol.7, N6. P. $136-141$.

5. Miroshnichenko, I.S. Splat-quenching from the melt / I.S. Miroshnichenko. M.: Metalurgy, - 1982. - 167p. (in Russian).

6. Bashev, V.F. Nonequlibrium crystallization and structure transitions at treatment microwiwes from alloys Fe-C and Fe-C-Si / V.F. Bashev // Physics of Metals and Metallography. - 1983. - Vol. 55, N 2. - P. $331-336$.

7. Herlach, D. Crystal nucleation and dendrite growth of metastable phases in undercooled melts / D. Herlach // J. of Alloys and Compounds. - 2011. - V. 5095. - P.513 - 517.

8. Curiotto, S. Effect of cooling rate on the solidification of Cu58Co42 / S. Curiotto, N.H. Pryds, E. Johnson, L. Battezzati // Mat.Sci. and Eng. - 2007.-A 449-451. - P.644 - 648.

9. Funke, O. Dendrite growth velisity in levitated undercooled nickel melts / O.Funke, G. Phanikumar, P.K. Galenko, L. Chernova, S. Reutzel, M. Kolbe, D.M. Herlach // J. of Grystal Growth. - 2006. - N297. - P. 211 - 222. 